\title{
Improved design of stone cutting machine based on disassembly
}

\section{analysis}

\author{
Ding Zhizhong ${ }^{1,}$ a, Yu Chao ${ }^{2, b}$ \\ ${ }^{1,2}$ College of Mechanical and Electrical Engineering, Hohai University, Changzhou, P. R. China \\ adingzz7814@sina.com, ${ }^{b} 1161051906 @ q q . c o m$
}

Key words: Electric tool, cutting machine, man-machine, improvement.

Abstract: Introducing stone cutting machine in short, and conducting an analysis through a decomposition and dismantlement of a product labeled Mod.4110B. Then come up with an eventual and ultimate modification concentrated on the additional shell, independent handle grip, more comfortable auxiliary grip, less incorporation of harmful powder, with as more probable factors as possible taken into consideration. Propose ideas for solutions to the existing problems, with further improvement of practical design.

\section{Introduction}

Foremost, cutting machine is widely used in the cutting field of constructional materials like terrazzo, marble, granite, those sorts of hard and brittle of non-metals with a manifest advantage of high efficiency and high quality but together with potential working risk, because it's working with manual control and high power. Thus some general problems, like noise and vibration, powder harmful to respiratory system, probable occupational diseases, shared by other electronic tools with a high power exists in terms of both product design and man-machine coordination. Once these latent problems can't be wiped out, they would in turn have an impact to the security of worker and the efficiency of their work. Afterwards, this paper is working to better combine the improvement of cutting tools and product design so as to prevent the possibly appearing problems from causing bad results and then the machine can accordingly serve better for both society and individual.

Secondly, this passage is going to conduct a comprehensive and exhaustive analysis through decomposing and dismantling the model conduct, SPECTRUM Mod.41110B. Through this reverse decomposition, the internal spatial structure, parts distribution, and working status of these parts can be understood with depth, thus some possible improvement could be posed.

\section{Product dismantling and use scenario analysis}

Decomposed product: SPECTRUM Mod.41110B. Basic parameter: showing in table 1.

TABLE1. BASIC PARAMETER OF PRODUCT

\begin{tabular}{|c|c|c|c|}
\hline Input power & $1380 \mathrm{w}$ & Chamfer cutting & none \\
\hline No-load speed & $11000 \mathrm{R} / \mathrm{min}$ & Blade diameter & $110 \mathrm{~cm}$ \\
\hline maximum & $30 \mathrm{~cm}$ & Net weight & $2.7 \mathrm{~kg}$ \\
\hline Protection level & $\varnothing / \mathrm{II}$ & Insulation level & $\mathrm{E}$ \\
\hline
\end{tabular}


Decomposition process (Fig1): separate the product into three sections, the working point, working body, and the working tail.

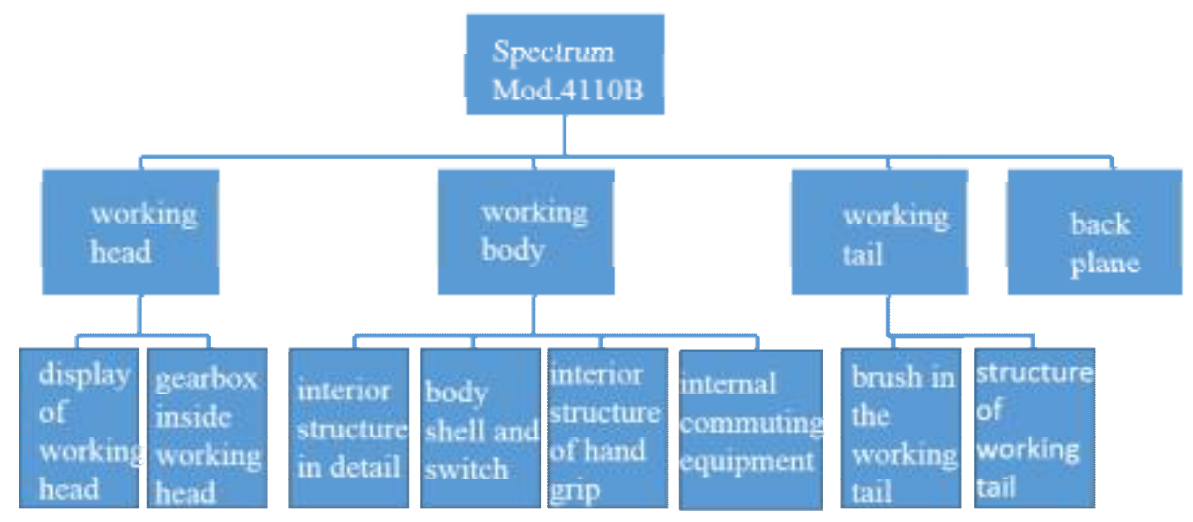

Figure1.dismantle flow

The decomposing subsequence: the product is decomposed space-orderly form the working head to the end of the machine. Working head is divided into three parts, including the silver-gray metal, black metal shaft and the shaft housing. Figure 2 is the head part of the marble machine. With a simple structure of the metal shell, there're four bolts inside the shell making it closely integrated with the fuselage. Axis, extending from the center axis and the blade is mounted by two thick irons and a central bolt. (Fig3)

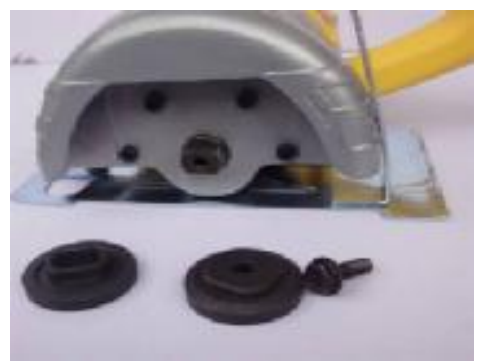

Figure2. Display of working head

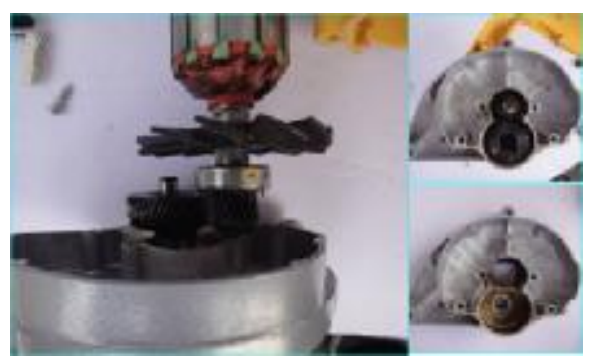

Figure3. Inside of working head

Part of the internal structure of the fuselage (Fig4): there is an interior motor. The blade is installed on the outermost bearing, combined with the rotation direction of the blade and the direction of inclination of the bearing, something can be seen that blade wind is blowing motors, it can be inferred that the motor is an internal heat evacuation. The main body, and some controller means including the switch controller, commentator, brushes, etc. These units are compactly embedded within the body, but there are also a lot of ribs landing in the interior housing. (Fig5)

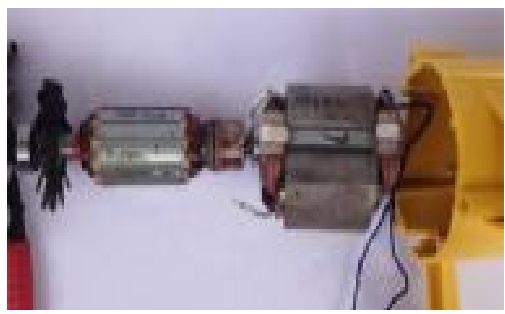

Figure4. Interior structure in details

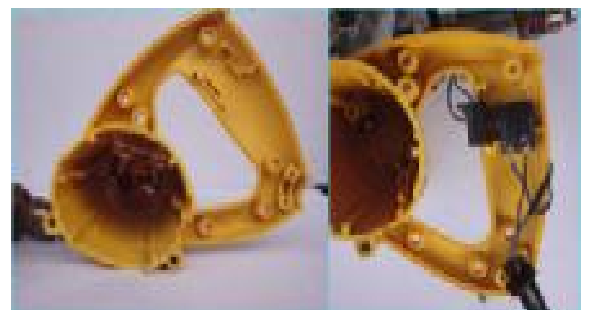

Figure5. Body shell and switch

What can be seen from the main body handle of the internal structure of the space is that the hand section with a considerably occupation of space, place only the switch and wires connected to the switch inside the switch and the switch is connected to the wire. In the portion corresponding to the index finger placed switch and plastic dust cover, the pressure line control the wire to avoid pulling the wires to cause electrical problems. Thus concluded that the shape of the handle is entirely due to design of the appearance, also depending on how comfortable for people during use, 
so that the handle can be highly variable can be easily summed up (Fig6) . Commentator is a section of DC permanent magnet series motor, serving to keep the motor continually rotating. (Fig7)

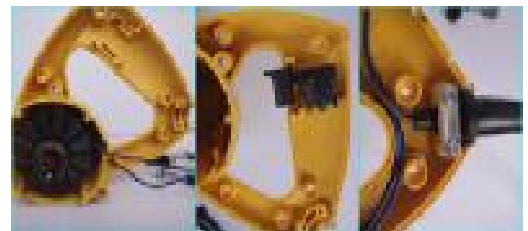

Figure6. Structure of hand grips

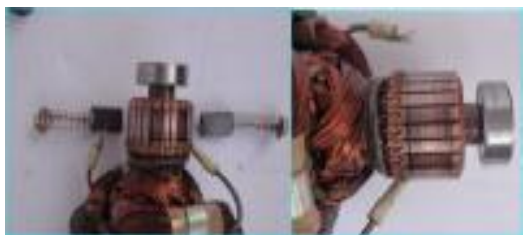

Figure7. Commuting equipment

What match with the commentator is carbon brushes in figure8. Brushless DC motor must be rectified to motivate, which is necessary for motor commutation. Brush has anti-electromagnetic interference capability, wearing resistance and good commutation ability. Marble machine rear fuselage (Fig9) is to provide space to accommodate some of the controller marble machine fuselage body and also set a number of cooling ducts. Finally, peripheral device on the machine plate show in figure10. Machine floor connected with the body through bolts, together with the front adjustable knob and the depth of cut can be adjusted when cutting. In addition the marble machine can be made sure to move forward in working and thus ensure the quality of operations.

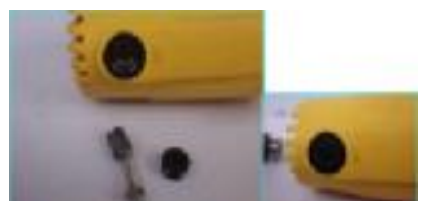

Figure8. Brush in the working tail

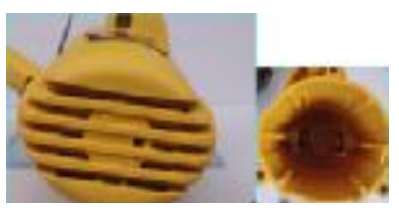

Figure9. Structure of working tail
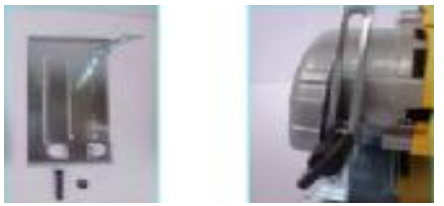

Figure10. Backplane

\section{The analysis of how it's operated}

Here is the demonstration of how the marble cutting machine is adjusting cutting depth (Fig11, 12). Following is the display of the use of the Mod. 4110B marble machine switches. From observing the design of the shape and the position of the switch, it can be easily to know how to use the switch but user may feel a little uncomfortable because of the shape and material of the lock button (Fig13, 14).

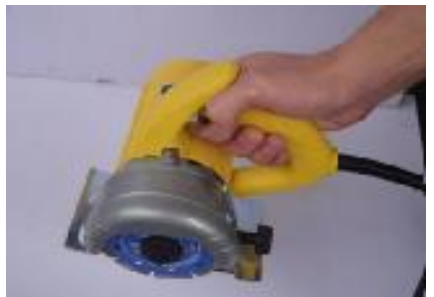

Figure11. Cutting posture

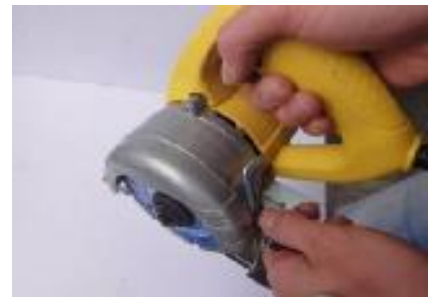

Figure12. adjusting the cutting

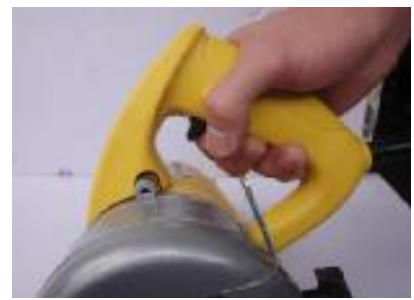

Figure13. Display of switch

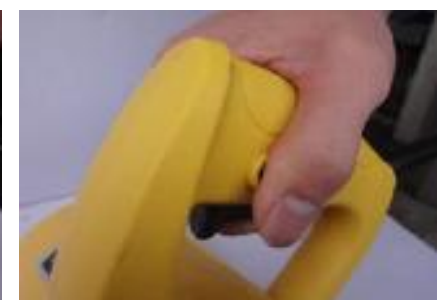

Figure14. Lock-on button

Whether it would be convenient to install the open edge is directly related to the convenience of using marble cutting machine. Basically operator has to change different edge according to the different cutting materials every time, thus having that procedure easy to learn and master is pretty necessary. While at the same time stability during use of the blade must be sure. Here is the demo of how the blade of spectrum Mod.4110B is installed. (Fig15)

From the analysis above, we had some improved points available after the decomposition. We have now roughly known about the structure working head, the work movement, body, operating handle, these parts, among which the tail of the machine and handle has a large adjustable space.

From materials analysis we find that marble machine shell use highly insulating materials, and wear-resistant, heat-resistant, electrical insulation and high strength comprehensive security performance is the primary objective for marble machine design. 


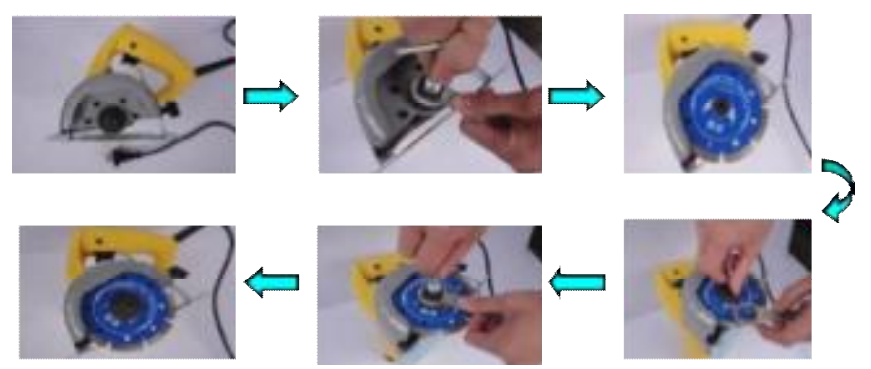

Figure15.flow chart of blade installment

\section{Consideration before improvement}

1. The shortcoming of stone cutting tools when using

The possibly problem during use mainly involves vibration, powder, and risk brought by the inappropriate operation when the tools are in non-work condition. The following passage is going to come up with respective solution according to these points.

This passage is working from the point of studying physical and mental occasion of human, plumbing the appropriate use method to reduce and decrease the vibration in the using process in terms of man-machine principle [1-2]. Thus an initial improved design could be generated to ameliorate the vibration [3-4].

2. Design concept in term of cutting machine itself

The design concept based on the open blade of the cutting machine. Users are thought to not use the tools during the work break and it's not worthwhile to cut the power because it will cost more energy plus its open blade, so some wrong operation is more likely to happen and thus cause risks. Secondly speaking, the open blade of the non-work tools is in direct touch with the working table, so it's wise to put and catch it with a light ease otherwise its blade will get hurt.

In order to solve this problem, a shield added between the blade and the anti-leaching shield and close that shield when not using it so that the safety risks can be largely reduced. Secondly the additional shield can protect the blade by avoiding a direct touch with the working facet. Though there is a more operation of shield opening, it's an essential account in terms of the safe use.

3. The design concept based on the security risk of single-hands operation

With a series of advantage like a compact body and structure, a light quality, even a lightly higher vibration and long-time operation would render fatigue to man's body especially arms, then following some muscle illness. What's worse, the accuracy and precision and working quality would get influenced by vibration. Plus the cutting direction is the right circle, which means that the machine would generate a large scale of beat if met even a light block, in other words if the operator can't have a good command on the vibration generated by the cutting tools, there would be a potential risk.

Working from the previous experiment, there is a highly variable parts of the marble machine handle, thus handle can be designed as one according to the shape of working arm of human. Plus, with a front lateral auxiliary handle, two hands can be utilized to control that tool, which largely increase the safety. Also that front auxiliary handle makes it easy to be portable when in transportation so that it reflect a sense of humanistic in terms of use or a carrying state. Specific auxiliary handle lies in front of the main handle, a smooth grip, which is beneficial for both comfort and combination with the overall structure.

4. The design concept based on the improvement of comfort during use

The operator will feel arm numb, hand fatigue, when the vibration is slightly larger during use. Furthermore long-time use will cause some arm muscle diseases and corresponding soft issue injury, so how to reduce the damage of the vibration of cutting tools is quite worth an in-depth study. 
A solution according to both processing part of handle's man-machine design and matching section of hand fitting is raised that firstly taking advantage of not having many structure in the inner of the handle so having handle shape changed can make it useful and comfortable to operate for up to $90 \%$ users. Then an encapsulated process to the handle can thus enhance the comfort.

5. The design concept based on how to reduce the powder generated during operation

Marble machine will generate a large amount of dust which is harmful to human so the amount is a significant standard to measure the machine cause user will be working in an atmosphere full of dust thus the eyesight and other breathe system will get hurt. That's why the amount of dust should be designed to cut to a lower degree than the existing degree.

To stop the large area spreading of dust, a means of a wider large working head has to be proposed. Normally countries have a strict regulation on the amount of dust in the process of using tools. Usually electronic tools would be in accordance to the country standard but in order to reduce the dust generation, a solution, using a much wider working head to stop dust from a large area spreading of dust, has thus to be proposed.

6. Design concept based on ergonomic to improve gripping comfort

The user has a variably different requirement of the optimal grip span selection because factors, like gender length of hands, width of hands power peak, are not the same, but through seriously strict design there can be an optimal angle for user [5-8].

Pistol grip style handle and intermediate shank handle requires an optimum angle [8]. According to ergonomic principles, the angle of the handle arm joint edges with hand held tool connection substantially parallel with the axis of the handle angle and the articulated arm should be a line between the best angle $[3,5,6]$. This angle is typically the angle of $70^{\circ}$, then the operator can both save effort, and acquire a high precision[1, 3, 5].

Besides the need to handle design marble machine within range of the manual control, so you can better operate the marble machine in a more comfortable way. The angle of the handle and the handle diameter should be combined with user's size.

Study abroad about the material shows that the polyethylene propylene handle provides a comfortable, good gripping surface [5,7]. With a flexible surface of the handle you will feel comfortable. Griping force exerted on the plastic handle requires less force than griping the aluminum handle with the same diameter $[3,7]$.

The size of the pistol handle switch trigger has a large influence on the force of the user's hand $[3,5]$. When increasing the size, the force can be reduced to a level which user can easily apply to. Moreover trigger's design should match the action range of hands, and the finger's working areas.

\section{The eventual and ultimate design}

In abstraction of what have been raised above, this passage choose the relatively mature solution that having an auxiliary handle to match the traditional marble machine; however we now have another problem.

A small handle can serve marble machine well during normal cutting, but the angle of handle will change when adjusting the cutting depth or cutting angle, plus the short and small handle, user will not be able to achieve the previously expected outcome but only increase the using difficulty, which is contradicted to the original point, so such a solution is going to be abandoned.

The interdependence of the auxiliary handle and a then amplification would be a key solution in order to break the limitation of the inconvenience of griping handle when cutting in different depth or angle, so that the need to cut in a diversity of both depth and angle would be fulfilled. 
The enlarged Auxiliary handle has a tubular shape, from the tail of working body to the left of the main handle, and the extended auxiliary handle allows the user to release the left hand, making them not confined to narrow work space, also the user won't suffer from hand fatigue. Since there is a large work space for activity, user can adjust where their hand grip according to the specific personal habits and work environment. This will not only increase productivity, but also avoid muscle disease caused by strong vibration.

The cutting machine is in idle condition and the protective cover can cut the contact between the blade and the outside, to avoid the damage by a misuse of blade rotation (Fig16).Now the machine is in working condition and the protective cover is open so that the machine can normally work (Fig17). The machine now is in a state after an adjustment of cutting depth when the cover can protect the exposed part, both reducing the spread of dust and guaranteeing the safety of users (Fig18).

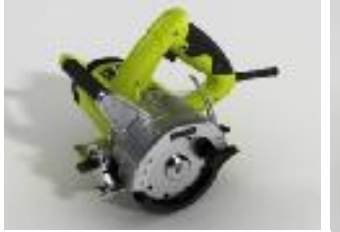

Figure16. demo1

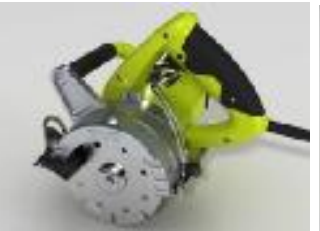

Figure 17. demo2

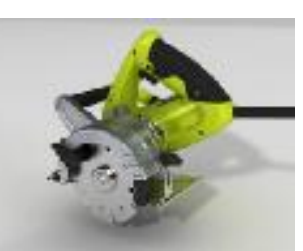

Figure18. demo3

The starting point is to meet the needs ot product design, comprising numan needs, the needs of society and natural needs [10]. Product design is not only the substantial transformation of demands but also the relationship transformation of demands. So whether the purpose of designing or the factors that have an impact on product design, are inseparable from the environmental system composed by human, society, objects, and nature [10-12]. In easy words, it's what appears to be when product is related to the users and the using occasion, including the mutual interaction and the accordingly formed interacting situation give rise to the inspiration of designer so that they can make contribution to the overall design industry [10].

From the terms of simulation and holding the mock in reality, auxiliary handle grip played a relatively good supporting effect, but there were also many design flaws, such as the working head lying on the left side of the machine, has an impact on the eyesight and vision of the operator to some degrees, and this doesn't affect the left-handed the least slightly, but make it more comfortable. Given the crowd of right-handed is in relatively large, we can take into a further consideration to locate that working heat on the right side of the product, so that bringing more convenience to the majority of people. (Fig19, 20)

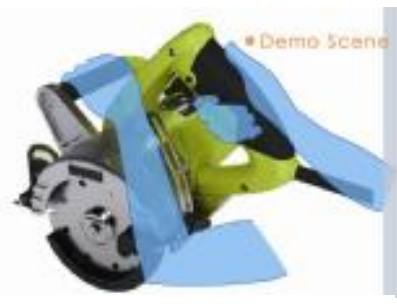

Figure19. Demo of use

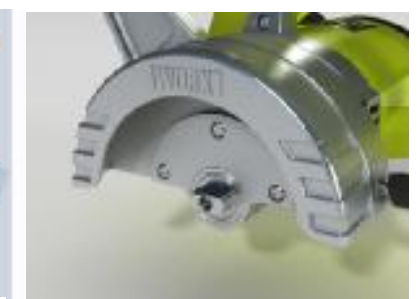

Figure20. Part in details

\section{Conclusion, summary and extension}

Passage above has posed solution to these problems, an additional to largely decrease the risk of open blade, an arm-shaped handle plus two hands to operate when necessary to improve the security of single-handed operation, a modified handle shape together with a encapsulating processing to conform the comfortable use of up to $90 \%$ users, and a wide large working head to stop the large 
areas spreading of dust. Moreover, an improvement according to the ergonomic has also applied to the handle design.

In term of the whole improved design program of the cutting machine, the protection of the blade has been solved out, and some accidents caused by some misuse can be completely avoided. Talking about the auxiliary handle, it can give rise to the most use of the handle and also be portable to take and transport. Both the two function can already be applied to the cutting machine.

This passage has mainly focused on the two aspects; security and comfort, there are obviously other points failing to be taken into consideration more or less. We can easily foresee that the cutting machine is going to do a couple of progresses, like the integration with battery, which can be a developing trend of the whole industry. The very lastly, I strong believe that day won't be far off.

\section{Reference}

[1] Huang Dengjun, Analysis of Tremor Frequency of Hammer and Adaptability of the Acceptable Range, [J], China Packaging Industry, 2014, 14, pp. 4.

[2] Jiang Nan, Yan Zhongkang,, Luo Xifeng, Serial Design of Household Electric Tool [J], Packaging Engineering. 2008,5, pp. 115-117.

[3] Zhu Qiang, Qin Yongyuan, Ergonomics and Power Tools, [J], Electric Tool, 2005,2, pp. 1-10

[4] Xia Rulong., The Research on Grip Comfort of the Tool Handles Based on Pressure Distribution, [D], Zhejiang, Zhejiang University of Technology, 2013.

[5] Qin Zhaosheng, Human Engineering Technology and Tools design, [J], Electric Tool, 2002, 2, pp. 5-8.

[6] Wu Chunlin, Research on Green Design Technique of Power Tools, [D], Suzhou, Suzhou University, 2011.

[7] Lu Haibing, Use of Human Engineering and Electronic Technology in the Field of Power Tools [J], Coastal Enterprises and Science \& Technology. 2007, 2, pp. 80-81.

[8] Wu Shan, Small Household Electric Drill Product Design, [D], Nanchang, Nanchang University, 2013.

[9] Ma Tianyuan, Deng Yanning, Amenity Analysis of Handle Tools for Automotive Maintenance, [J], Mechanical Engineer. 2013,2, pp. 80-81.

[10] Zhao Jianguo, Product' Situation Constructing and Semanteme Creating in Design, [D], Hunan, Hunan University, 2009.

[11] Yu Fan, Yin Runyuan, System Analysis of Bionic Design, [J], Packaging Engineering. 2008, 6, pp. 141-144.

[12] Xu Xihua, Cultural Essence of Product Designing, [J], Journal of Zhejiang University (Humanities and Social Sciences), 2002, 4, pp, 117-123. 